\title{
First year results from the HAWC observatory
}

\author{
Sabrina Casanova for the HAWC Collaboration ${ }^{1,2, a}$ \\ ${ }^{1}$ Institute of Nuclear Physics Krakow, ul.Radzikowskiego 152, 31-342 Poland \\ ${ }^{2}$ Max Planck Institute for Nuclear Physics Heidelberg, Saupfercheckweg 1, 69117 Germany
}

\begin{abstract}
The High Altitude Water Cherenkov Observatory is an all-sky surveying instrument sensitive to gamma rays and cosmic rays from $100 \mathrm{GeV}$ to $100 \mathrm{TeV}$. With its $2 \mathrm{sr}$ instantaneous field of view and a duty cycle of $>95 \%$, HAWC is carrying out an unbiased survey of the Northern sky and is monitoring known flaring sources and searching for transients. HAWC operation began mid-2013 with a partially-completed detector. The array was terminated in 2015 . We here summarize the status of the observatory, and highlight its first scientific results, resulting from the first year of data taking after completion of the detector. In particular, we will present the HAWC map of the sky at tens of $\mathrm{TeV}$.
\end{abstract}

\section{Introduction}

The High Altitude Water-Cherenkov Telescope (HAWC) is an air-shower array facility, designed to study $\mathrm{TeV}$ gamma rays by observing the air-shower particles, which, produced by the $\mathrm{TeV}$ photons in the high atmosphere, arrive in the detector. HAWC data taking started in August 2013 while still under construction. HAWC construction was terminated at the beginning of 2015. The complete detector consists of 300 Water Cherenkov detectors (WCDs) spread on a 22000 square meter area. Every WCD is a $4.5 \mathrm{~m}$ high tank made of steel with a diameter of $7.3 \mathrm{~m}$, and contains 200,000 liters of purified water. At the bottom of the tank there are three 8-inch photomultipliers (PMTs) arranged in a triangular layout, plus one high quantum-efficiency 10-inch PMT at the center of this triangle. The photomultipliers detects the Cherenkov light produced by the air shower particles in each WCD unit. HAWC is continuously recording signals from each WCD and seeking for multiple coincident signals or events. The data are reconstructed event per event, each event having between 20 and 50 channels hit. The shower direction is reconstructed by collecting and comparing the arrival times of the shower particles in the different WCDs hit by the shower. The detector is calibrated by means of a laser calibration system which delivers light pulses to all the WCD in the array using optical fibers. This laser signal guarantees the timing and charge calibration of the individual PMTs, which are crucial for an accurate reconstruction of the air shower. The shower fronts are fitted with sub-nanosecond residuals, resulting in an angular resolution as good as 0.2 degree for the highest energy events. The charge deposits on each PMT channel is used for energy estimations and for gamma/hadron discrimination through algorithms based on core location and the pattern of the energy deposition in the array

ae-mail: sabrinacasanova@gmail.com 
- including muon identification. HAWC registers over 20,000 cosmic rays per second, generating 2 TB of data every day.

Its wide field-of-view, with an instantaneous aperture of $2 \mathrm{sr}$, covers more than $15 \%$ of the sky so that the detector is exposed to two-thirds of the sky during a 24-hour period. This makes HAWC an ideal survey and monitoring observatory, complementary to the pointing instruments at $\mathrm{TeV}$ energies, the imaging air Cherenkov telescopes (IACTs), such as H.E.S.S., MAGIC, VERITAS, and the upcoming Cherenkov Telescope Array (CTA). While the IACTs reach their best sensitivity at about 1 $\mathrm{TeV}$, the HAWC observatory is particularly sensitive to photons of energy above several TeVs. In fact, HAWC sensitivity curve to steady sources peaks at $10 \mathrm{TeV}$ and furthermore is constant for extended sources up to three degrees across [1]. These two key features make HAWC an ideal instrument to discover and study Galactic gamma-ray sources produced by the interactions of the highest energy cosmic rays. In the following section we will review the HAWC map of the sky at tens of TeV obtained by the HAWC Collaboration after collecting one year of data.

\section{Scientific results}

Primary science goals of the HAWC observatory are searching for the origin of the highest energy cosmic-rays, studying transient phenomena, such gamma-ray binaries and gamma-ray bursts [2], monitoring active Galactic Nuclei, searching for dark matter signals [3] and studying more exotic topics such as primordial black holes [4].

A year after completion of the observatory, the HAWC Collaboration announced a new map of the sky in very high energetic (VHE) gamma rays [5]. The sky map in Fig.1 shows several astrophysical objects along the plane of our galaxy, many of which have not been observed previously. Among the extended galactic sources observed by HAWC it is worth mentioning the extended emission discovered close to two famous middle-age closeby pulsars, Geminga, and PSR B0656+14, associated with the Monogem ring (see Fig.2). The two HAWC sources are likely pulsar wind nebulae powered by ultrarelativistic electrons accelerated at the termination shocks, where the powerful pulsar winds encounter the interstellar medium. The HAWC discovery of extended $\mathrm{TeV}$ emission from the directions of both Geminga and the pulsar PSR B0656+14 offers a unique chance to investigate the VHE particle populations in our Galactic neighbourhood, shedding light on the origin of these particles and understanding the local particle transport at the highest energies, which is not constrained by any cosmic ray measurements.

Another region of great astronomical interest mapped by HAWC is the Cygnus region, the brightest region in $\mathrm{GeV}$ and $\mathrm{TeV}$ gamma-rays in the Northern sky (see Fig.2). The region was previously mapped at $\mathrm{TeV}$ energies with the Milagro instrument [6-8] and one of the two Milagro sources in Cygnus, MGRO J2019+37, was later spatially resolved by Veritas in two sources, which still lack a clear counterpart at lower energies [9]. The study of the non-thermal radiation from the Cygnus region, dominated by one of the brightest and closest star forming region in our Galaxy, Cygnus X, is crucial for understanding the origin of cosmic rays in the Galaxy and the relationship between star formation and acceleration of particles. Detailed studies of the different $\mathrm{TeV}$ sources observed by HAWC are currently under way.

The new non-thermal map of the universe to the highest energies also includes two famous active galactic nuclei, Markarian 501 and Markarian 421, the closest BL Lac objects known, whose lightcurves are shown in Fig.3 and Fig.4, respectively. Thanks to the high significances of the detections, $33 \sigma$ for MrK421 and $23 \sigma$ for Mrk501 it was possible to extract their daily flux level. The HAWC light curve of Mrk421, including 387 selected transits, represents the first daily monitoring of a TeV blazar with the sensitivity to actually detect flaring on single transits. Frequent high states of 


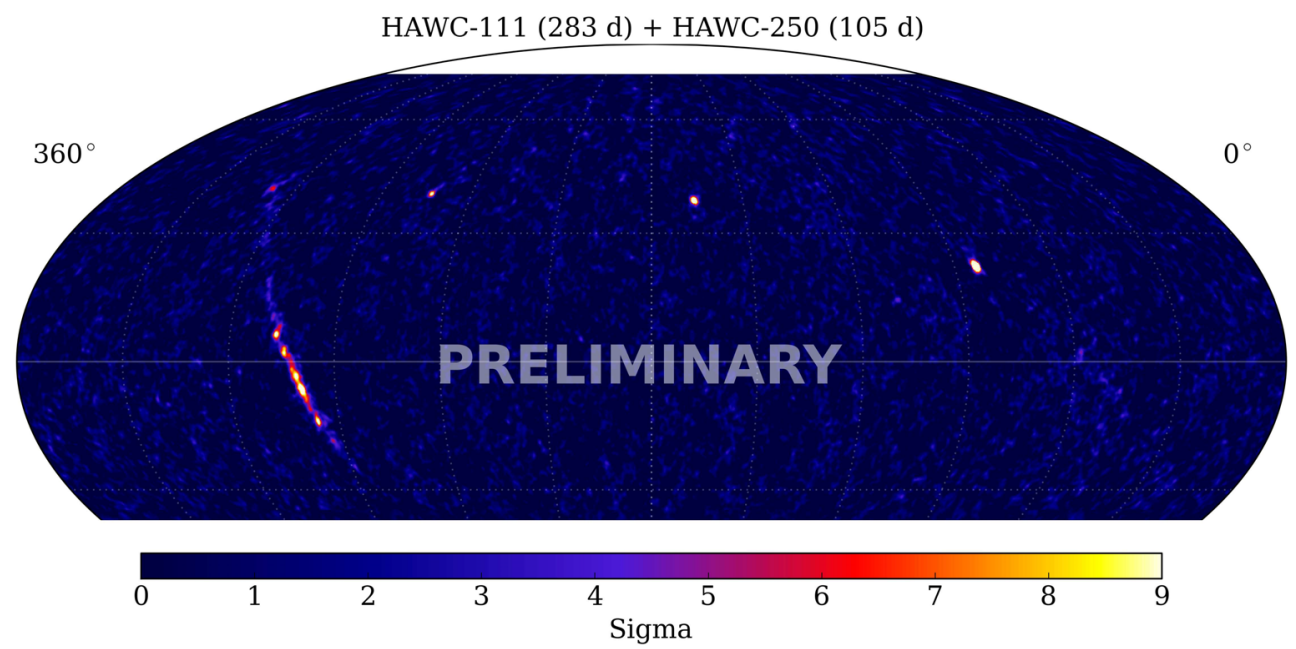

Figure 1. HAWC first year all-sky map in celestial coordinates. Several astrophysical objects along the plane of our galaxy, many of which have not been observed previously, are visible. The three point sources are Mrk 501, Mrk 421 and the Crab Nebula. The HAWC results were announced in a press release during the April 2016 APS meeting [5].
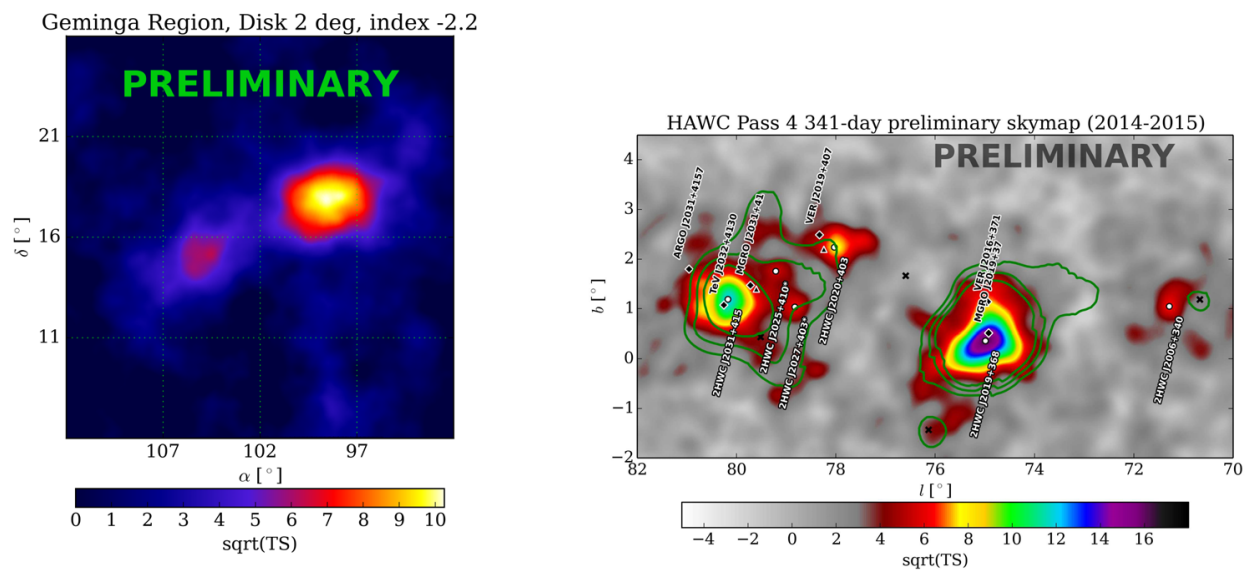

Figure 2. (Left Panel) HAWC excess map of the Geminga and PSR B0656+14 regions. (Right Panel) Excess map of the Cygnus region, dominated at lower energy by one of the brightest and closest star forming region in our Galaxy, Cygnus X, where a high density in gas, pulsars and supernova remnants create an incredible environment for stars to be born. 


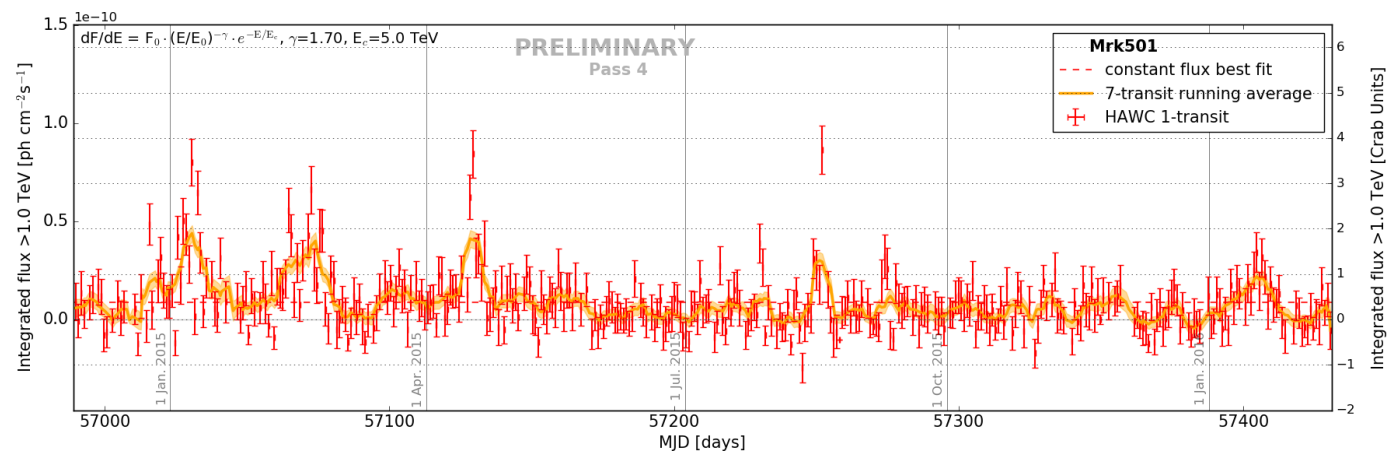

Figure 3. The lightcurve of the active Galactic Nucleus, Markarian 501 between November 262014 and February 122016 is shown. The fluxes are calculated assuming a spectral index of 1.7 and an exponential cut-off at $5 \mathrm{TeV}$. Episodes of flaring activity, with twice the Crab flux are evident.

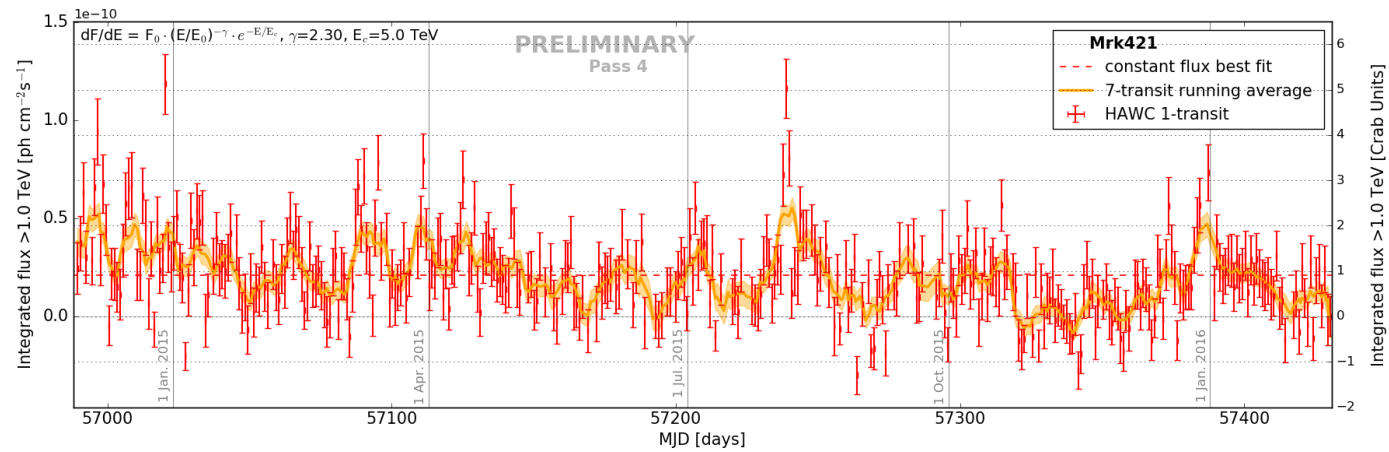

Figure 4. The lightcurves of the active Galactic Nucleus, Markarian 421 HAWC light curve of the BL Lac object Mrk 421 spans from November 2014 to February 2016 and shows several flaring states, in which the TeV flux of Mrk 421 was stronger than that of the Crab.

fluxes larger than 1 or 2 Crab have been observed. Recently, HAWC, FACT and Swift-XRT reported the joint observation of a four day activity of Mrk 421, which reached $2 \mathrm{Crab}$ units above $1 \mathrm{TeV}$ [10]. Mrk501, whole lightcurve is presented in Fig.4, was observed recently by HAWC while it was extremely bright, the two-day flare starting on April 6th 2016 reaching a flux 2.2 times that of the Crab [11]. This confirms that HAWC has the capability to provide timely alerts to the astronomical community.

Gamma-Ray Bursts (GRBs) have been detected up to several GeV by Fermi-LAT. However none of the $\mathrm{TeV}$ ground-based experiments has ever detected one yet. The main problem is that they are unpredictable, and due to the reduced duty cycle of the IACTs, it is difficult to detect them. The large duty cycle of HAWC ( $>90 \%$ ), together with its large field of view makes HAWC the ideal experiment to detect GRBs at TeV energies. HAWC is expected to detect a rate as high as $1.6 \mathrm{GRB}$ per year. One of the brightest GRB in the last years happened while a small fraction of the detector 

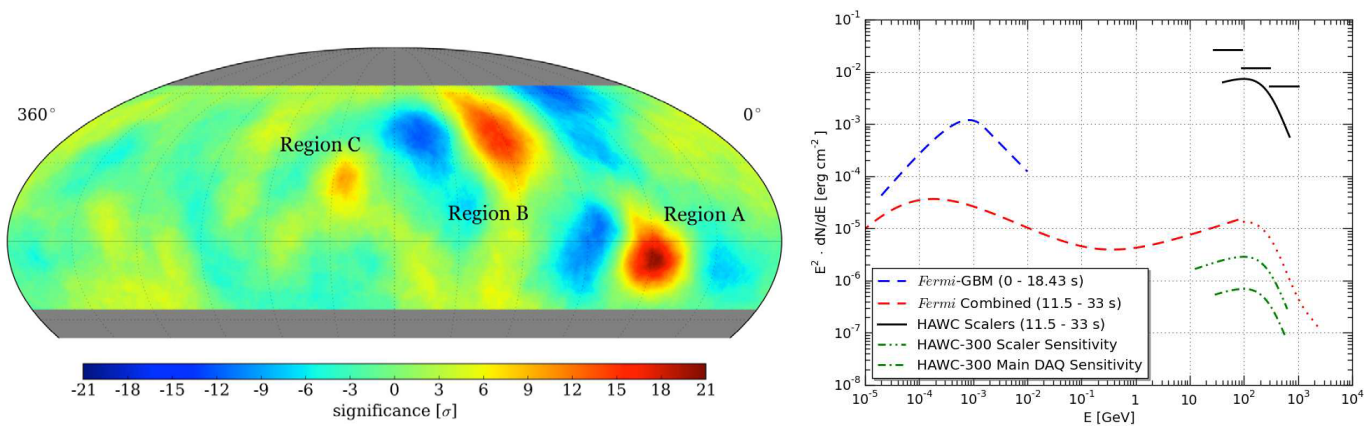

Figure 5. (Left Panel) HAWC cosmic-ray skymap. The large-scale structures have been subtracted to emphasize the three regions with enhanced cosmic-ray flux.(Right Panel) HAWC upper limits on GRB130427A. Together with the complete HAWC sensitivity and the flux measured by Fermi. The sensitivity for the complete detector (HAWC300) is shown for comparison.

(HAWC30) was taking data, and only the scaler data acquisition system (DAQ) was active. The burst was not detected, and the corresponding upper limits were derived (see right panel of Fig. 5 [12]).

The anisotropy of the arrival direction of the cosmic-rays, which was first detected by Milagro [13], and later on confirmed by other experiments like IceCube [14], has been also observed with HAWC [15]. The left panel of Figure 5 shows the cosmic ray sky map obtained with 113 days of HAWC-100 (HAWC detector with $100 \mathrm{WCD}$ ) data. It contains $5 \times 10^{10}$ cosmic-ray events with a median energy of $1.9 \mathrm{TeV}$, and a median angular resolution of $1.2 \mathrm{deg}$. The map shows the significance computed by using a $10 \mathrm{deg}$ smoothing angle. There are 3 regions with an excess of cosmic-rays above $5 \sigma$ level. Region A and B were already reported by Milagro experiment, and region $\mathrm{C}$ was recently announced by the ARGO-YBJ collaboration [16]. The origin of the cosmic-ray anisotropy is unclear. HAWC will shed some light on its origin by measuring the spectrum, flux, and variability of the cosmic-ray anisotropy.

\section{Conclusions and outlook}

Thanks to its nonpointed observations of photons up to energies of tens of $\mathrm{TeV}$, its large field of view, and its high duty cycle HAWC is the perfect instrument for surveying and monitoring the TeV gammaray sky. One year after completion of the array, the HAWC collaboration has presented the first map of the Galactic Plane at multi-TeV energies. HAWC continuously monitors the VHE sky. Recently it has detected Markarian 501 in a flaring state and promptly launched an alert to the astronomical community.

\section{Acknowledgments}

We acknowledge the support from: US National Science Foundation (NSF); US Department of Energy Office of High-Energy Physics; The Laboratory Directed Research and Development (LDRD) program of Los Alamos National Laboratory; Consejo Nacional de Ciencia y Tecnologia (CONACyT), Mexico; Red de Fisica de Altas Energıs, Mexico; DGAPA-UNAM, Mexico; and the University of Wisconsin Alumni Research Foundation. Sabrina Casanova acknowledges the support from the Polish National Science Center under the Opus Grant UMO-2014/13/B/ST9/00945. 


\section{References}

[1] A.U. Abeysekara, R. Alfaro, C. Alvarez, J.D. Álvarez, R. Arceo, J.C. Arteaga-Velázquez, H.A. Ayala Solares, A.S. Barber, B.M. Baughman, N. Bautista-Elivar et al., Astroparticle Physics 50, 26 (2013), 1306.5800

[2] A.U. Abeysekara, J.A. Aguilar, S. Aguilar, R. Alfaro, E. Almaraz, C. Álvarez, J.d.D. ÁlvarezRomero, M. Álvarez, R. Arceo, J.C. Arteaga-Velázquez et al., Astroparticle Physics 35, 641 (2012), 1108.6034

[3] A.U. Abeysekara, R. Alfaro, C. Alvarez, J.D. Álvarez, R. Arceo, J.C. Arteaga-Velázquez, H.A. Ayala Solares, A.S. Barber, B.M. Baughman, N. Bautista-Elivar et al., @ @ stylePhys. Rev. D90, $122002(2014), 1405.1730$

[4] A.A. Abdo, A.U. Abeysekara, R. Alfaro, B.T. Allen, C. Alvarez, J.D. Álvarez, R. Arceo, J.C. Arteaga-Velázquez, T. Aune, H.A. Ayala Solares et al., Astroparticle Physics 64, 4 (2015), 1407.1686

[5] M. Hui, The HAWC Galactic Plane Survey, in APS April Meeting Abstracts (2016)

[6] A.A. Abdo, B. Allen, D. Berley, E. Blaufuss, S. Casanova, C. Chen, D.G. Coyne, R.S. Delay, B.L. Dingus, R.W. Ellsworth et al., @ @ styleApJ658, L33 (2007), astro-ph/0611691

[7] A.A. Abdo, B. Allen, T. Aune, D. Berley, E. Blaufuss, S. Casanova, C. Chen, B.L. Dingus, R.W. Ellsworth, L. Fleysher et al., @ @ styleApJ688, 1078-1083 (2008), 0805.0417

[8] A.A. Abdo, U. Abeysekara, B.T. Allen, T. Aune, D. Berley, E. Bonamente, G.E. Christopher, T. DeYoung, B.L. Dingus, R.W. Ellsworth et al., @ @styleApJ753, 159 (2012), 1202.0846

[9] E. Aliu, T. Aune, B. Behera, M. Beilicke, W. Benbow, K. Berger, R. Bird, A. Bouvier, J.H. Buckley, V. Bugaev et al., @ @ styleApJ788, 78 (2014), 1404.1841

[10] A. Biland, D. Dorner, R. Lauer, J. Wood, B. Kapanadze, A. Kreikenbohm, The Astronomer's Telegram 9137 (2016)

[11] A. Sandoval, R. Lauer, J. Wood, The Astronomer's Telegram 8922 (2016)

[12] A.U. Abeysekara, R. Alfaro, C. Alvarez, J.D. Álvarez, R. Arceo, J.C. Arteaga-Velázquez, H.A. Ayala Solares, A.S. Barber, B.M. Baughman, N. Bautista-Elivar et al., @ @ styleApJ800, 78 (2015), 1410.1536

[13] A.A. Abdo, B.T. Allen, T. Aune, D. Berley, S. Casanova, C. Chen, B.L. Dingus, R.W. Ellsworth, L. Fleysher, R. Fleysher et al., @ @ styleApJ698, 2121 (2009), 0806.2293

[14] R. Abbasi, Y. Abdou, T. Abu-Zayyad, M. Ackermann, J. Adams, J.A. Aguilar, M. Ahlers, M.M. Allen, D. Altmann, K. Andeen et al., @ @ styleApJ746, 33 (2012), 1109.1017

[15] A.U. Abeysekara, R. Alfaro, C. Alvarez, J.D. Álvarez, R. Arceo, J.C. Arteaga-Velázquez, H.A. Ayala Solares, A.S. Barber, B.M. Baughman, N. Bautista-Elivar et al., @ @ styleApJ796, 108 (2014), 1408.4805

[16] B. Bartoli, P. Bernardini, X.J. Bi, I. Bolognino, P. Branchini, A. Budano, A.K. Calabrese Melcarne, P. Camarri, Z. Cao, R. Cardarelli et al., @@stylePhys. Rev. D88, 082001 (2013), 1309.6182 